\title{
Synthetic Jet Performance for Different Axisymmetric Cavities Analyzed with 3-D Lattice-Boltzmann Method
}

Pierre E. Sullivan, Hongbin Mu, Qingdong Yan, and Wei Wei

Version Post-print/accepted manuscript

Citation Mu H, Yan Q, Wei W, Sullivan PE. Synthetic Jet Performance for (published version) Different Axisymmetric Cavities Analyzed with Three-Dimensional Lattice-Boltzmann Method. AIAA Journal. 2018 Feb 28:1-7.

Doi: $\underline{10.2514 / 1 . J 056921}$

Publisher's Statement

Copyright (C) 2017 by the American Institute of Aeronautics and Astronautics, Inc. All rights reserved. All requests for copying and permission to reprint should be submitted to CCC at www.copyright.com; employ the ISSN 0001-1452 (print) or 1533$385 X$ (online) to initiate your request. See also AIAA Rights and Permissions www. aiaa.org/randp.

Read More: https://arc.aiaa.org/doi/abs/10.2514/1.J056373

How to cite TSpace items

Always cite the published version, so the author(s) will receive recognition through services that track citation counts, e.g. Scopus. If you need to cite the page number of the author manuscript from TSpace because you cannot access the published version, then cite the TSpace version in addition to the published version using the permanent URI (handle) found on the record page.

This article was made openly accessible by $U$ of $T$ Faculty.

Please tell us how this access benefits you. Your story matters. 


\title{
Synthetic Jet Performance for Different Axisymmetric Cavities Analyzed with 3-D Lattice-Boltzmann Method
}

\author{
Hongbin $\mathrm{Mu}^{*}$ \\ Beijing Institute of Technology, Beijing 100081, China \\ University of Toronto, Toronto, Ontario M5S 3G8, Canada \\ Qingdong Yan, ${ }^{\dagger}$ and Wei Wei ${ }^{\dagger}$ \\ Beijing Institute of Technology, Beijing 100081, China \\ and \\ Pierre E. Sullivan ${ }^{\S}$ \\ University of Toronto, Toronto, Ontario M5S 3G8, Canada
}

\section{Nomenclature}

$$
\begin{aligned}
& D=\text { oscillating driver diameter, } \mathrm{mm} \\
& V_{\mathrm{c}}=\text { cavity volume, } \mathrm{mm}^{3} \\
& d \quad=\quad \text { nozzle diameter, } \mathrm{mm} \\
& l_{\mathrm{n}} \quad=\quad \text { nozzle length, } \mathrm{mm} \\
& f \quad=\quad \text { oscillating driver frequency, } \mathrm{Hz} \\
& U_{\text {inlet }}=\quad \text { inlet velocity of cavity, } \mathrm{m} / \mathrm{s} \\
& U_{\text {inlet_max }}=\text { maximum inlet velocity of cavity, } \mathrm{m} / \mathrm{s} \\
& U=\text { phase-averaged axial jet velocity at nozzle exit plane, } \mathrm{m} / \mathrm{s} \\
& U_{\mathrm{cl}}=\text { centerline jet velocity at nozzle exit plane, } \mathrm{m} / \mathrm{s} \\
& U_{\text {cl_max }}=\text { maximum centerline jet velocity at nozzle exit plane, } \mathrm{m} / \mathrm{s} \\
& \bar{U}_{\mathrm{ts}} \quad=\quad \text { time-and-spatially averaged velocity at nozzle exit plane, } \mathrm{m} / \mathrm{s} \\
& \operatorname{Re}_{\bar{U}_{\mathrm{ts}}}=\quad \text { jet Reynolds number } \\
& S=\text { Stokes number for excitation frequency } f \\
& C=\text { constant for synthetic jet formation criterion }
\end{aligned}
$$

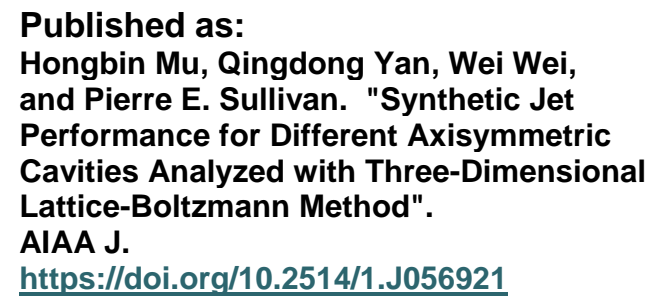

\footnotetext{
* Contact Author: PhD candidate, School of Mechanical Engineering, Beijing Institute of Technology and Department of Mechanical \& Industrial Engineering, University of Toronto, hongbin.mu@ mail.utoronto.ca.

${ }^{\dagger}$ Professor, School of Mechanical Engineering, Beijing Institute of Technology, yanqd@ bit.edu.cn.

* Associate Professor, School of Mechanical Engineering, Beijing Institute of Technology, weiweibit@ bit.edu.cn.

$\S$ Professor, Department of Mechanical \& Industrial Engineering, University of Toronto, sullivan@ mie.utoronto.ca.
} 


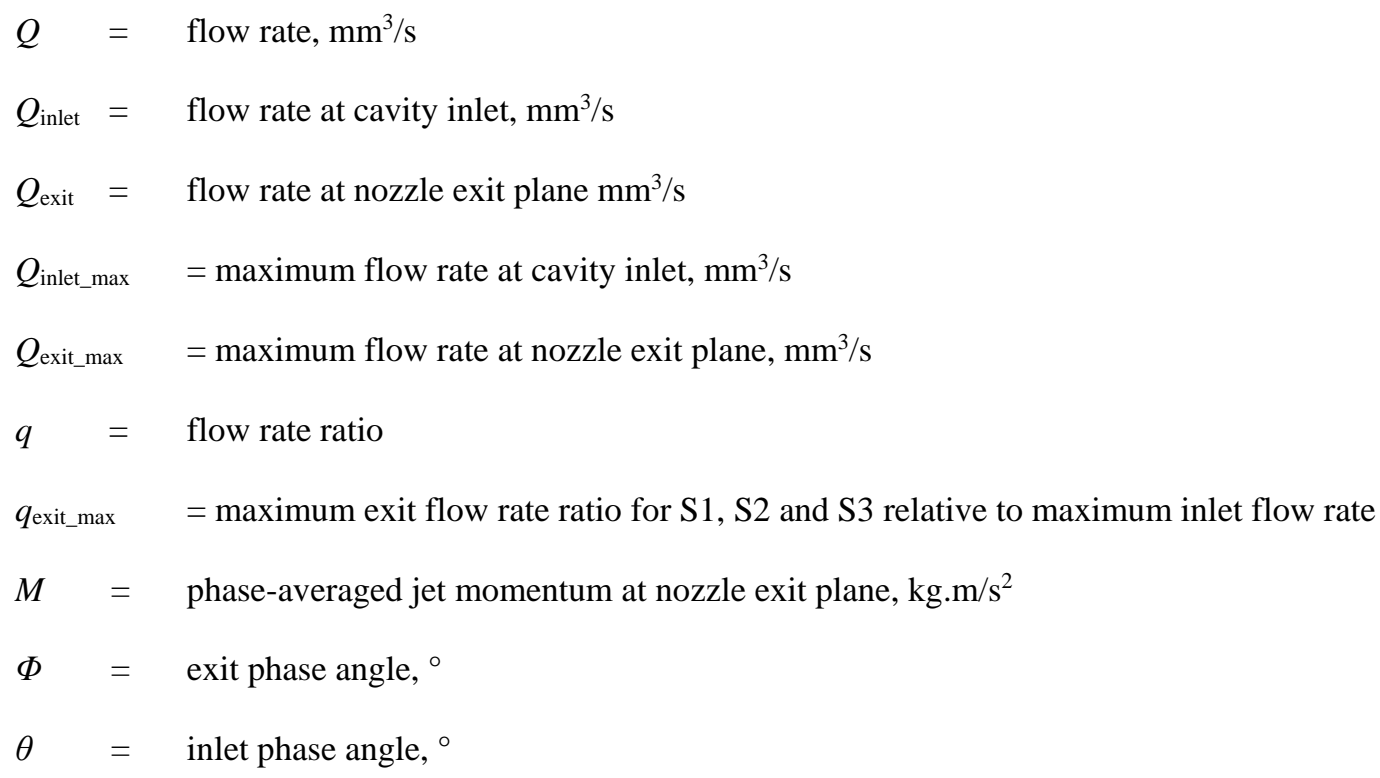

\section{I.Introduction}

A synthetic jet actuator is a zero-net-mass-flux device that imparts momentum and is useful for active flow control $[1,2]$. To study the influence of cavity shape on synthetic jet performance, this paper presents model results to validate previous experimental investigations that the synthetic jet is sensitive to cavity shape. Three axisymmetric synthetic jets with different cavity shapes, cylindrical, conical and contraction, were examined for jet performance, keeping other parameters constant such as cavity volume, nozzle length and nozzle diameter [3-5]. Using a 3-D Lattice Boltzmann method (LBM) with the Bhatnagar-Gross-Krook (BGK) collision models [6-8], the comparison of the three shaped cavities was conducted using the same sinusoidal velocity inlet boundary condition. Time-dependent synthetic jet simulations were carried out, the velocity and momentum profiles were illustrated and discussed, and the numerical simulations were validated against previous experimental data. The computed results confirmed that synthetic jet performance depend on cavity shape. The jet flow rate and momentum, relevant in flow control applications, decreased sequentially from the cylindrical to conical to contraction cavity.

\section{II.Numerical Method}

The synthetic jet actuators were modeled with axisymmetric cavities: cylindrical (S1), conical (S2) and contraction (S3) as well as their shaped cavity objects shown in Fig. 1. A cylindrical cavity shape (Fig. 1(a)) serves as the baseline case. Fig. 1 (b) and (c) show two other shapes selected for comparison: conical with a short cylindrical section and an axisymmetric contraction defined by a 5 th order polynomial. The different sections represent the different boundary 
conditions where the red line is the inlet with velocity profile, the black shows the no slip wall, and the blue shows the outlet with outflow condition [9].

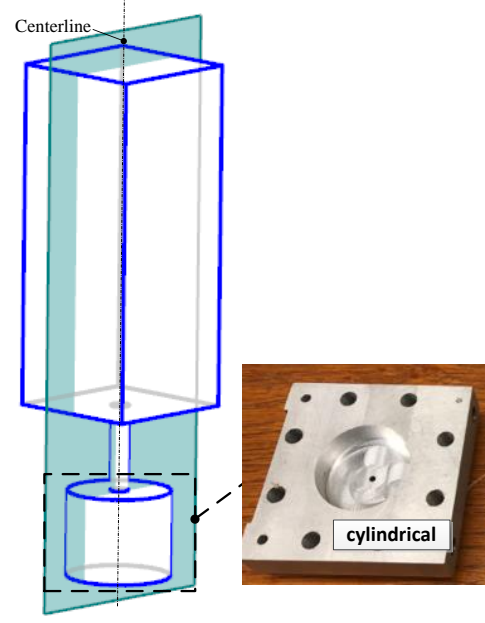

(a)S1

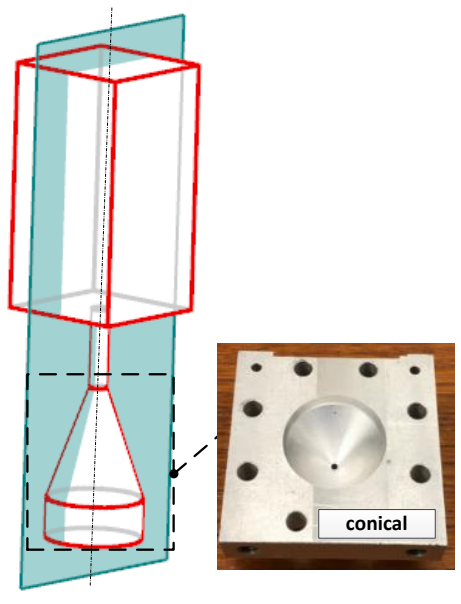

(b)S2

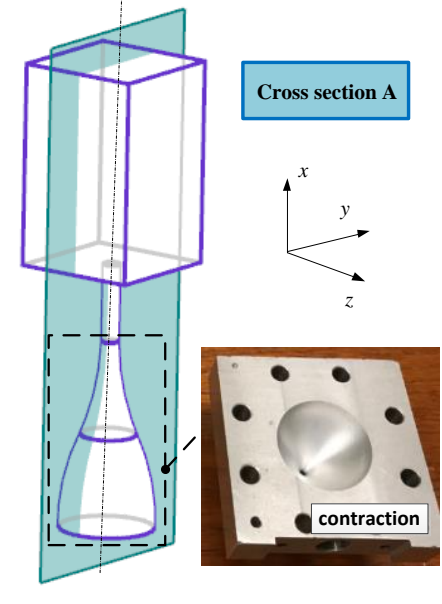

(c) S3

Fig. 1 Three synthetic jet actuator.

Table 1 compares the experimental and simulated geometries. Note that the radial length scale $y-z$ was shortened by having a smaller sized oscillating driver diameter and cavity volume.

Table 1 Comparison of experimental and simulated geometries

\begin{tabular}{lcc}
\hline \hline Parameters & Experiment & Simulation \\
\hline Oscillating driver diameter $D[\mathrm{~mm}]$ & 30.8 & 12 \\
Cavity volume $V_{\mathrm{c}}\left[\mathrm{mm}^{3}\right]$ & 9,313 & 1,150 \\
Nozzle diameter $d[\mathrm{~mm}]$ & 2 & 2 \\
Nozzle length $l_{\mathrm{n}}[\mathrm{mm}]$ & 10 & 10 \\
\hline \hline
\end{tabular}

To maintain geometric similarity among the three cavities, the following parameters were held constant: nozzle diameter $d=2 \mathrm{~mm}$, nozzle length $l_{\mathrm{n}}=10 \mathrm{~mm}$ and cavity volume $V_{\mathrm{c}}=1150 \mathrm{~mm}^{3}$, as shown in Table 1 . And the total scale of the 3-D computing domain is $30 d \times 8 d \times 8 d$. The cavity diameter was held constant at the diameter of the oscillating driver $D=12 \mathrm{~mm}$ and the cavity length $l_{\mathrm{c}}$ was increased in order to maintain constant $V_{\mathrm{c}}$ for S2 and S3. Unlike other computational studies [10,11], the downstream distance of the external region considered was only between $13 d-20 d$.

The frequency $f$ of the membrane sinusoidal oscillation velocity $U_{\text {inlet }}$ is

$$
U_{\text {inlet }}=U_{\text {inlet_max }} \sin (2 \pi f t)
$$


where $U_{\text {inlet_max }}$ is the oscillation magnitude of cavity inlet velocity. This boundary condition is a simple sinusoidal flow produced in the cavity by the motion of the diaphragm.

Matching test case parameters and synthetic jet performance were conducted based on the work of Feero et al. [12] with $\operatorname{Re}_{\bar{U}_{\mathrm{ts}}}=676$, and $S=22$. Vortex escape after the expulsion stroke was confirmed based on the synthetic jet formation criterion.

$$
\frac{\mathrm{Re}_{\bar{U}_{\mathrm{ts}}}}{S^{2}}>C
$$

where $C$ is a constant equal to 0.16 for 3-D nozzles.

To meet the formation criteria of synthetic jet actuator [13], $\operatorname{Re}_{\bar{U}_{\mathrm{Ls}}} / S^{2}=1.41$ and exceeds $C=0.16$ with $f=300 \mathrm{~Hz}$ [12] and meets the formation criterion for synthetic jets. Additional information on the model is found in [14].

\section{III.Results and Discussion}

Fig. 2 compares the phase-averaged flow rate and momentum profiles versus inlet phase angle at the nozzle exit for S1, S2 and S3. The maximum exit flow rate ratio $q_{\text {exit_max }}$ for S1, S2 and S3 relative to the maximum inlet flow rate $Q_{\text {inlet_max }}$ is

$$
q_{\text {exit_max }}=\frac{Q_{\text {exitmax }}}{Q_{\text {inlet_max }}}
$$

where $Q_{\text {exit_max }}$ is the maximum exit flow rate for $\mathrm{S} 1, \mathrm{~S} 2$ and $\mathrm{S} 3, Q_{\text {exit_S1_max }}, Q_{\text {exit_S2_max }}$ or $Q_{\text {exit_S3_max. }}$

The performance of the jet for flow control applications were quantified from the phase-averaged jet momentum calculated as

$$
M=\rho \int_{0}^{d / 2} 2 \pi U^{2} d r
$$

where $\rho$ is the density of the working fluid, air, at normal temperature.

In Fig. 2(a), it can be observed that the maximum exit flow rate, $Q_{\text {exit_max }}$, decreases sequentially from the cylindrical $\left(22,750 \mathrm{~mm}^{3} / \mathrm{s}\right)$ to conical $\left(19,250 \mathrm{~mm}^{3} / \mathrm{s}\right)$ to contraction $\left(16,020 \mathrm{~mm}^{3} / \mathrm{s}\right)$ cavity. As for the maximum exit flow rate ratio, $q_{\text {exit_max }}$, it varies from the cylindrical (0.404) to conical $(0.342)$ to contraction $(0.285)$ cavity when normalized with the maximum flow rate at the diaphragm. And from Fig. 2(b), it can be seen that the maximum 
momentum, in agreement with the velocity profiles, also decreases sequentially from the cylindrical $\left(1.76 \times 10^{-}\right.$ $\left.{ }^{4} \mathrm{~kg} \cdot \mathrm{m} / \mathrm{s}^{2}\right)$ to conical $\left(1.26 \times 10^{-4} \mathrm{~kg} \cdot \mathrm{m} / \mathrm{s}^{2}, 71.6 \%\right.$ of S1) to contraction $\left(7.40 \times 10^{-5} \mathrm{~kg} \cdot \mathrm{m} / \mathrm{s}^{2}, 42.0 \%\right.$ of S1) cavity. Cavity shape, for a constant cavity volume, impacts synthetic jet performance.

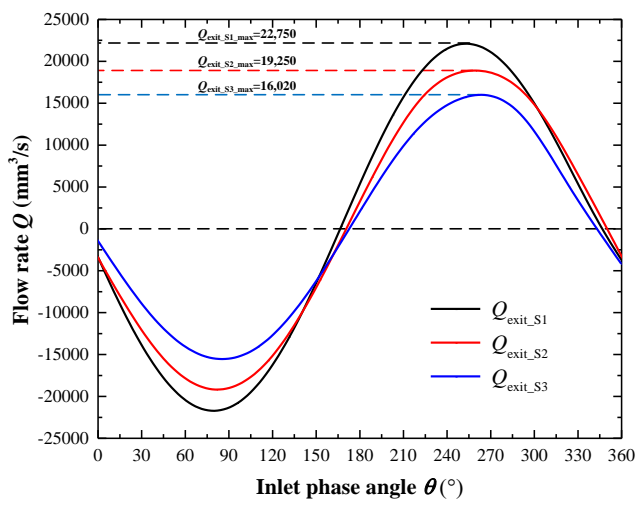

(a)jet flow rate

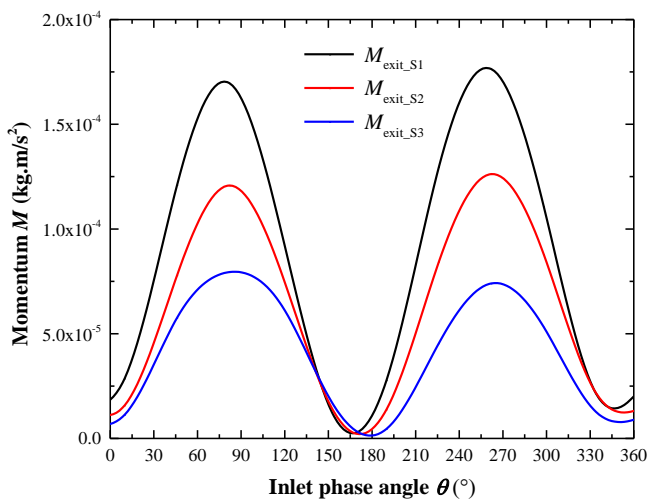

(b)jet momentum

Fig. 2 Phase-averaged flow rate and momentum profiles at the nozzle exit for S1, S2 and S3.

Fig. 3 compares the phase-averaged velocity profiles againt the inlet phase angle at the exit centerline in the ingestion and expulsion strokes. The exit phase angle $\Phi$ at $\Phi=90^{\circ}$ is maximum magnitude of expulsion velocity, while $\Phi=270^{\circ}$ is maximum magnitude of ingestion velocity.

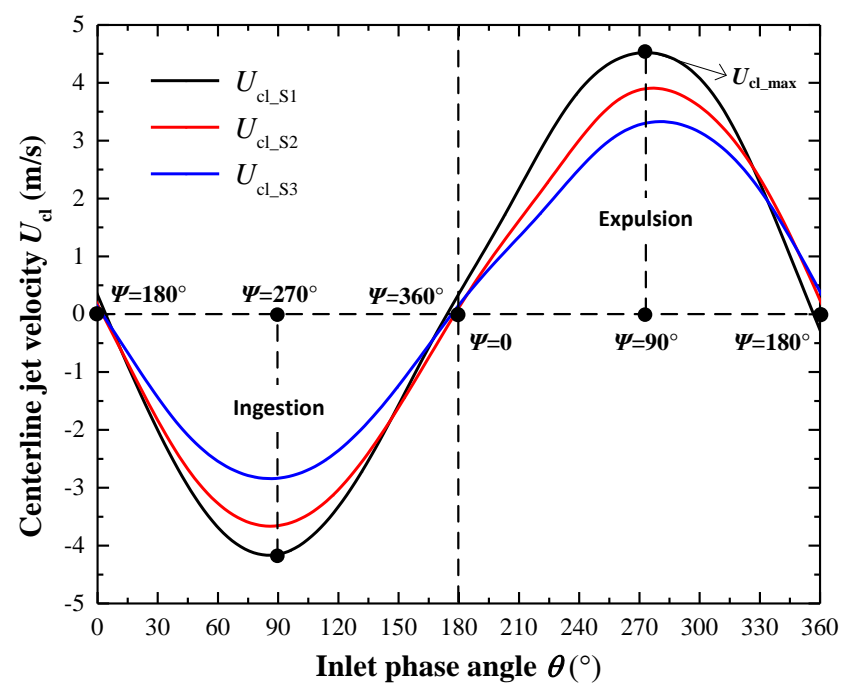

Fig. 3 Phase-averaged centerline jet velocity profiles at the nozzle exit for S1, S2 and S3. 
From Fig. 3, the exit phase angle $\Phi$ has a phase lag of roughly $180^{\circ}$, compared to the inlet phase angle $\theta$. The phase-averaged centerline jet velocity at the nozzle exit also shows periodic variation, similar to the inlet and exit velocity and flow rate profiles. Feero et al. [12] noted the maximum ingestion velocity at the centerline of the exit plane did not match the maximum expulsion velocity flow rate because inlet mass returns along the periphery of the jet. For the same surface conditions, the magnitude of the velocity is generally largest for S1 and slightly lower for S2, with S3 having the lowest velocities.
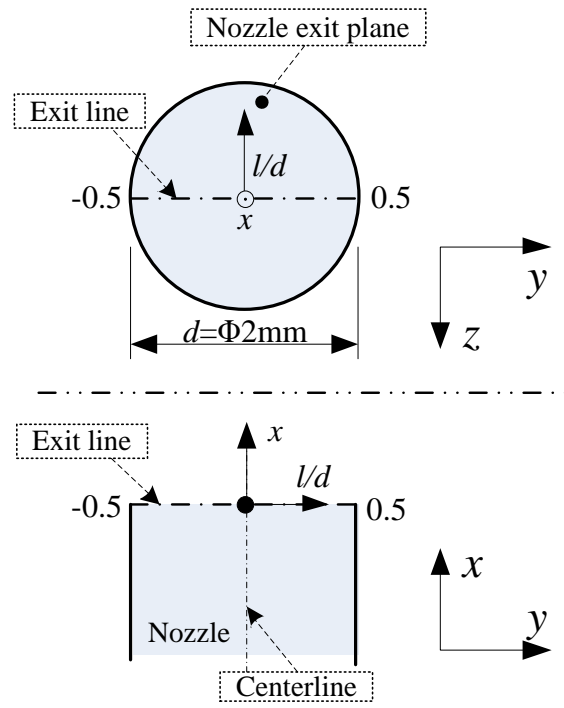

Fig. 4 Radial location of simulated jet velocity across the nozzle exit plane.

To examine the flow across the nozzle, the center of the nozzle exit plane in Fig. 4 and the non-dimensional modeled locations, $l / d$, are set between -0.5 to 0.5 . At $l / d=0$, the origin of the nozzle exit line is at the centerline.

Fig. 5 compares the normalized jet velocity at the nozzle exit line between the previous experimental results and the present work. Based on available simulated data at different inlet angles, the maximum expulsion velocity (exit phase angle $\left.\Phi=90^{\circ}\right)$ and ingestion velocity $\left(\Phi=270^{\circ}\right)$ were interpolated and compared with experimental results at $\Phi=90^{\circ}$ and $270^{\circ}$. Each curve was normalized by the maximum centerline jet velocity for S1, S2 and S3. 


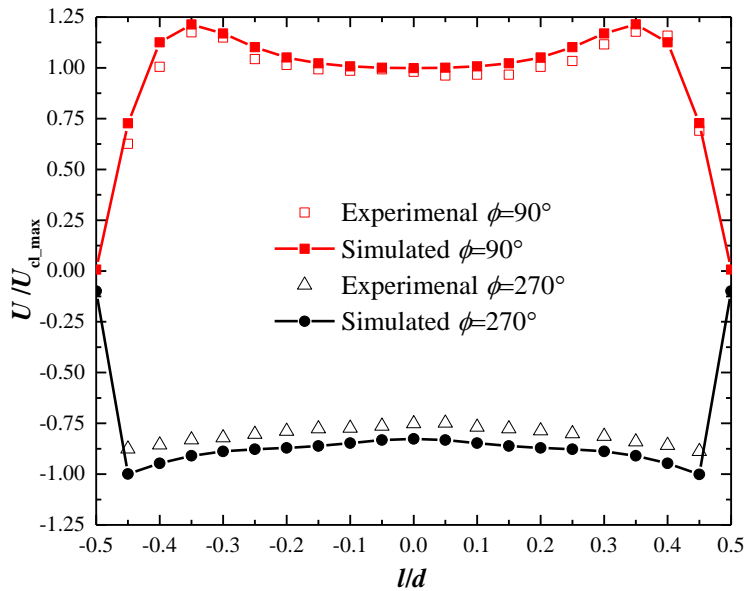

(a)S1

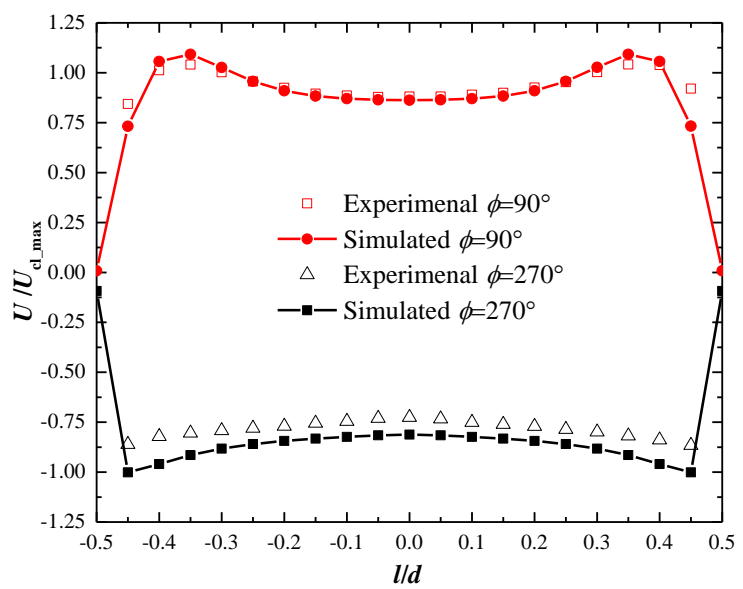

(b)S2

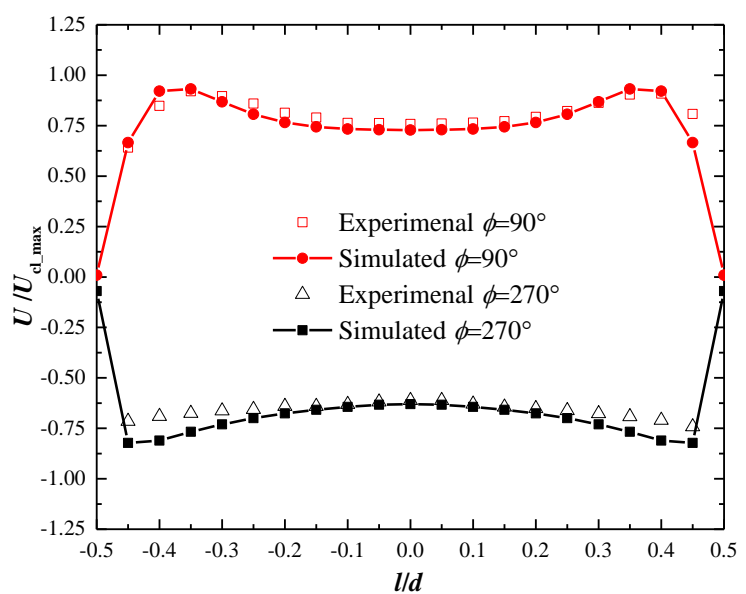

(c)S3

Fig. 5 Comparison of normalized jet velocity at the exit for different exit phase angles. 
Although the simulated models have a smaller oscillating driver diameter and cavity volume than the experiment, the structural parameters of the simulated and experimental nozzle are consistent. From Fig. 5, the numerical results of the three shaped cavities are all in good agreement with Feero et al. [12], particularly in the $l / d$ range from - 0.4 to 0.4. At $\Phi=90^{\circ}$, the average ratios of the computational variation with the experiments from $l / d=-0.4$ to $l / d=0.4$ are $4.44 \%, 2.21 \%$ and $3.85 \%$ for S1, S2 and S3 respectively, while the ratios at $\Phi=270^{\circ}$ are $9.88 \%, 11.3 \%$ and $6.69 \%$. It should be noted that the measurements were performed with hotwire anemometry and were thus unable to resolve reverse flow and were challenging at the ends of the nozzle $|l / d|=0.4-0.5$. As well, the experiments were performed $x / d=0.075$, where larger errors near the wall for S1, S2 and S3 are expected.

Velocity contours at cross section A, see Fig. 1, for different inlet phase angles are presented for the cavities S1, S2 and S3 in Fig. 6, Fig. 7 and Fig. 8.

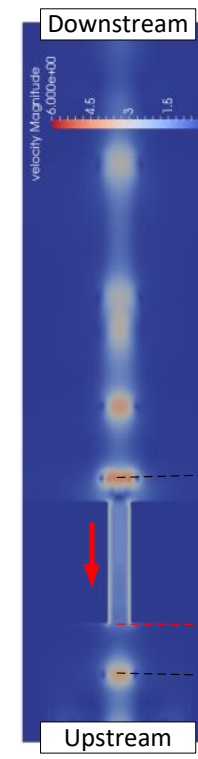

(a) $\theta=10^{\circ}$

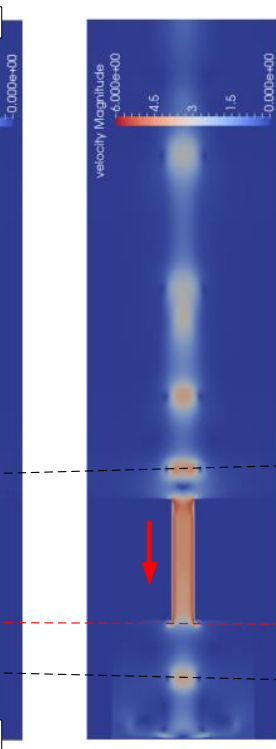

(b) $\theta=46^{\circ}$

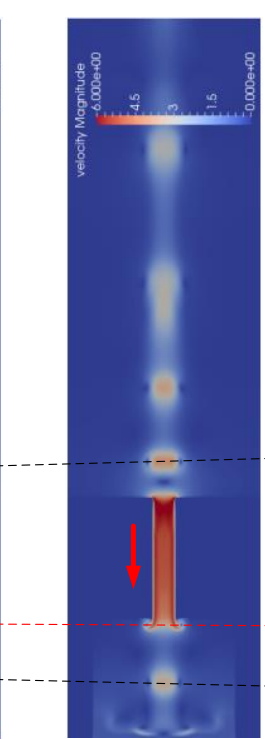

(c) $\theta=82^{\circ}$

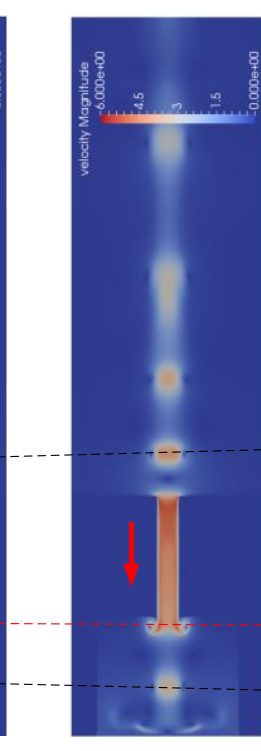

(d) $\theta=118^{\circ}$

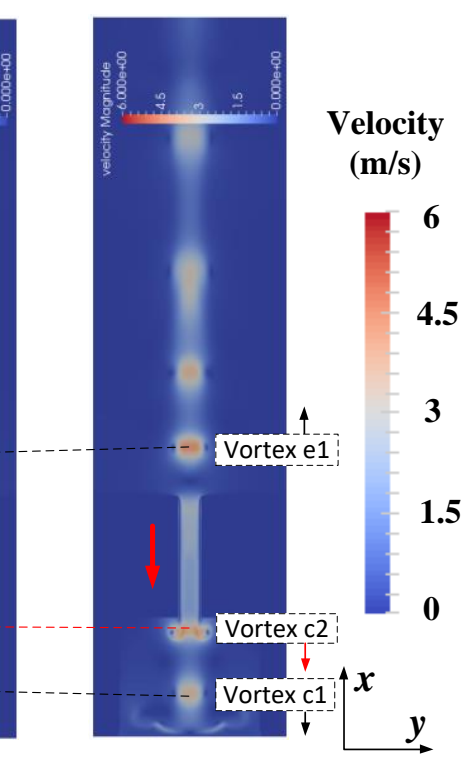

(e) $\theta=154^{\circ}$ 


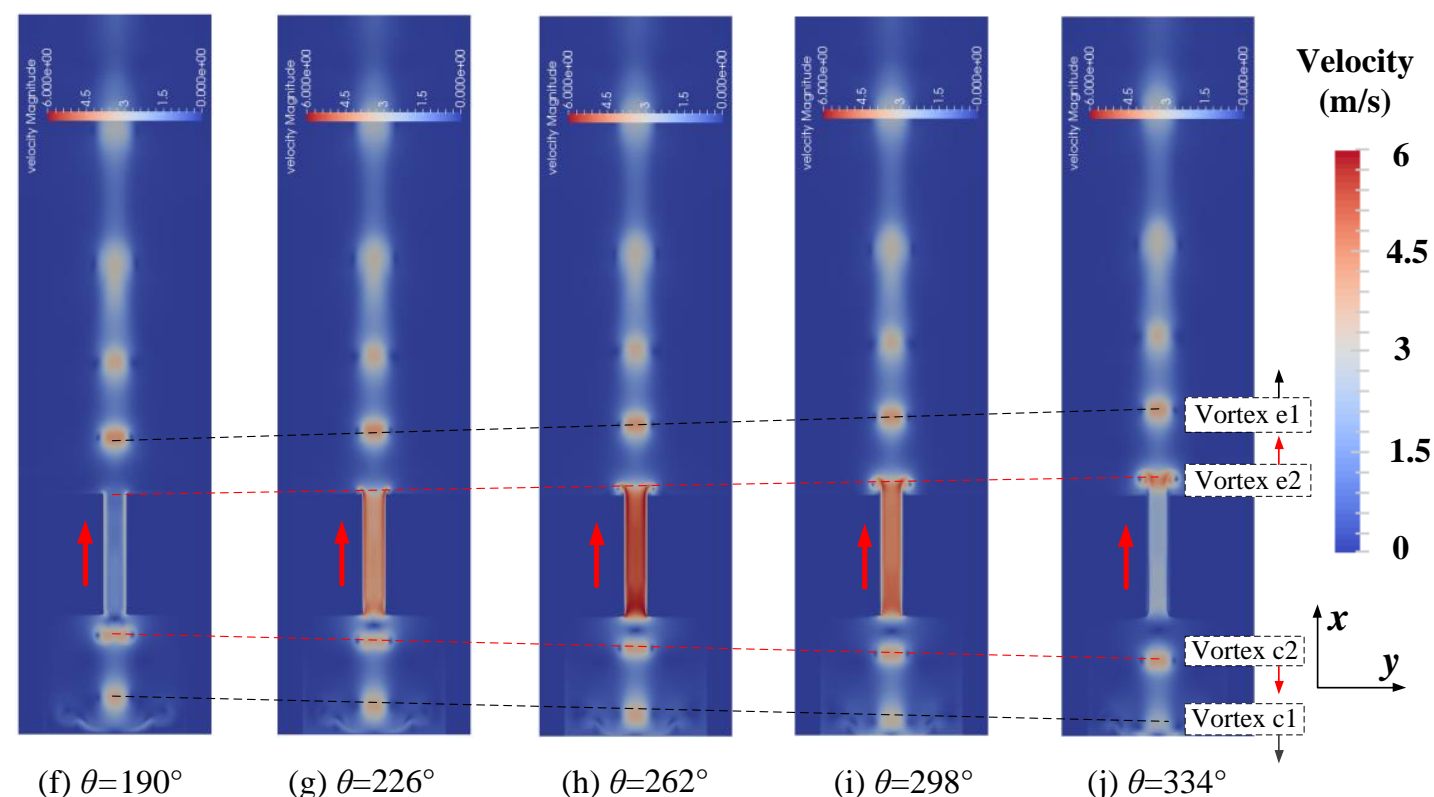

Fig. 6 Velocity contour at cross section A, S1.

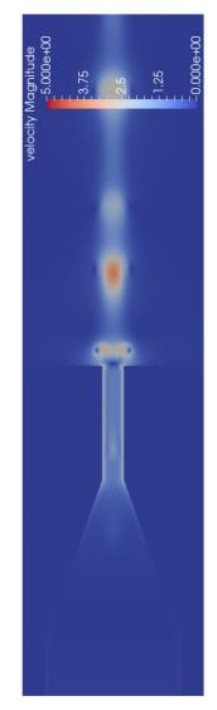

(a) $\theta=10^{\circ}$

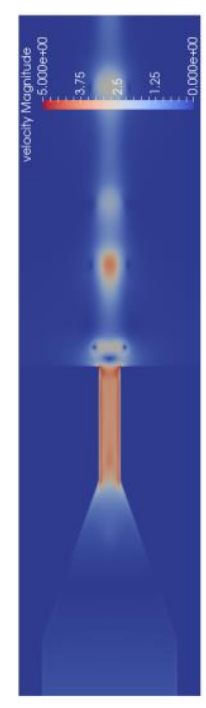

(b) $\theta=46^{\circ}$

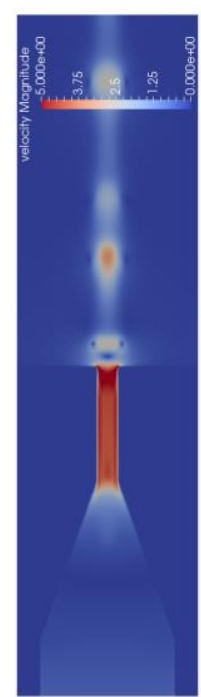

(c) $\theta=82^{\circ}$

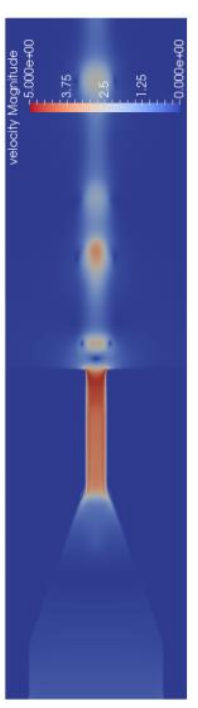

(d) $\theta=118^{\circ}$

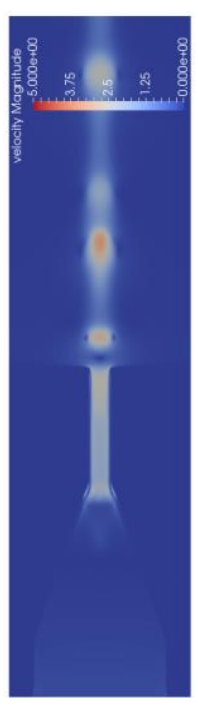

(e) $\theta=154^{\circ}$

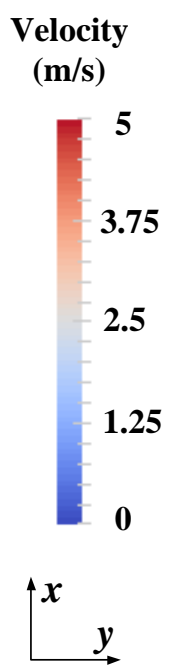




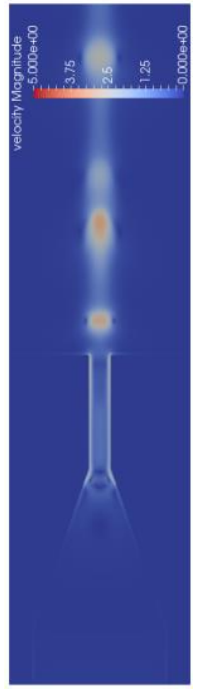

(f) $\theta=190^{\circ}$

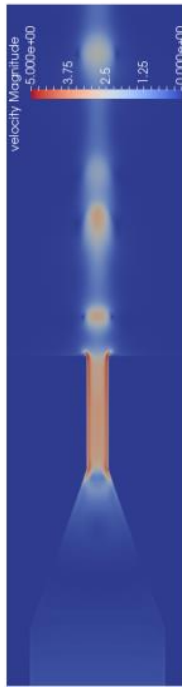

(g) $\theta=226^{\circ}$

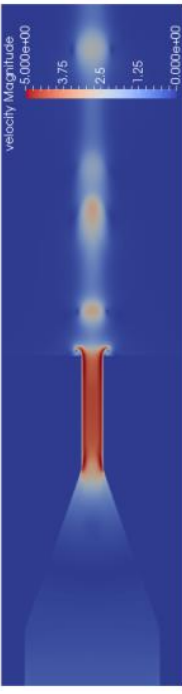

(h) $\theta=262^{\circ}$

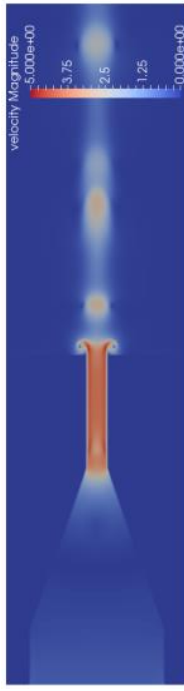

(i) $\theta=298^{\circ}$

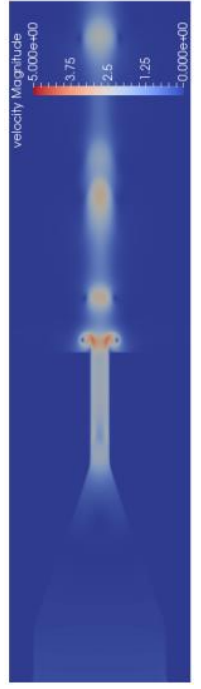

(j) $\theta=334^{\circ}$
Velocity

$(\mathrm{m} / \mathrm{s})$

5

3.75

2.5

1.25
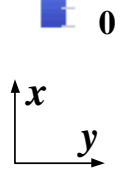

$\stackrel{y}{\longrightarrow}$

Fig. 7 Velocity contour at cross section A, S2.

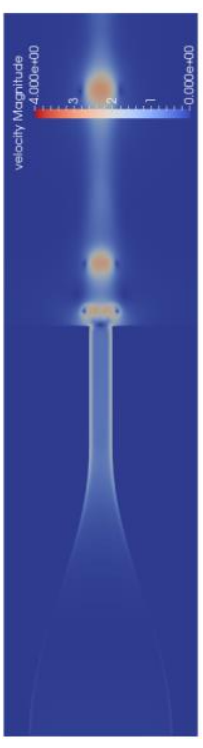

(a) $\theta=10^{\circ}$

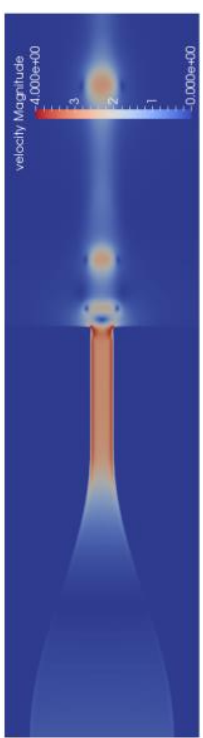

(b) $\theta=46^{\circ}$

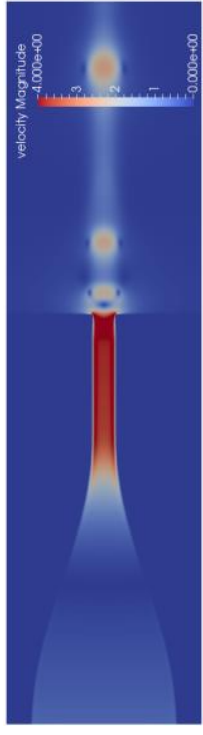

(c) $\theta=82^{\circ}$

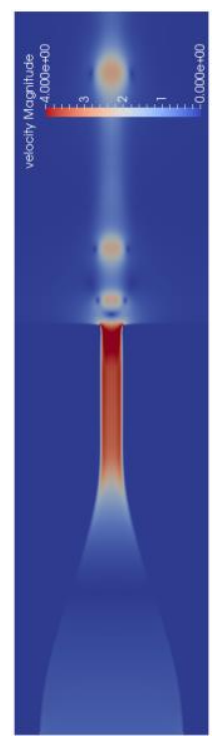

(d) $\theta=118^{\circ}$

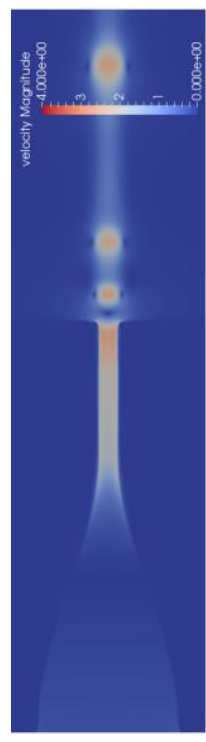

Velocity

$(\mathrm{m} / \mathrm{s})$

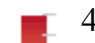

3

2

1

0

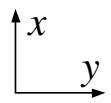

(e) $\theta=154^{\circ}$ 


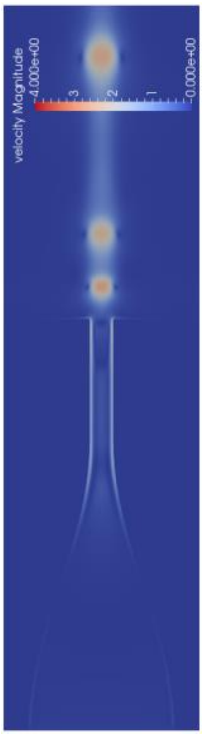

(f) $\theta=190^{\circ}$

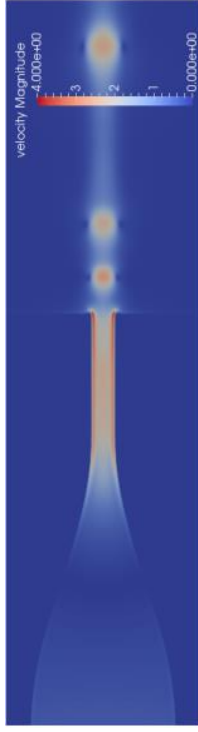

(g) $\theta=226^{\circ}$

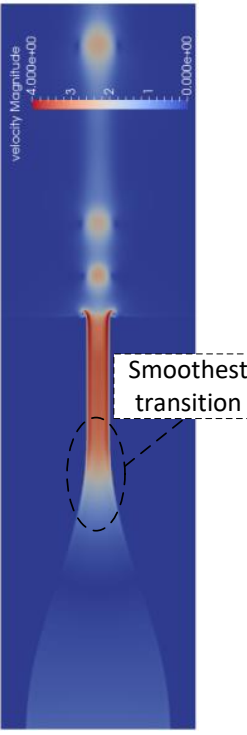

(h) $\theta=262^{\circ}$

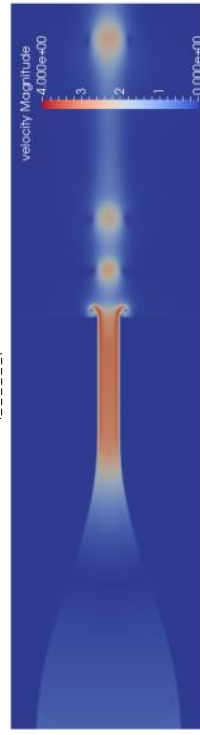

(i) $\theta=298^{\circ}$

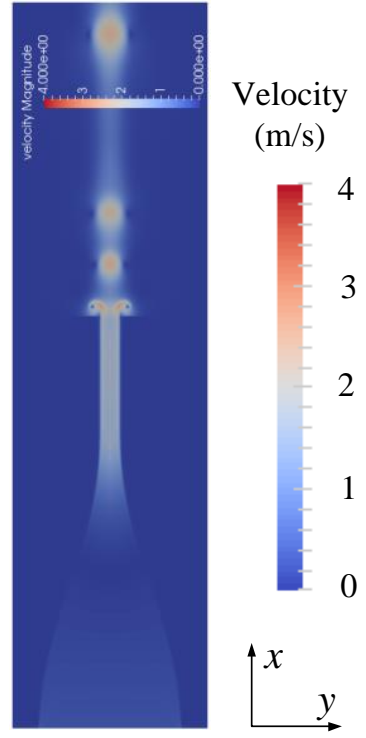

(j) $\theta=334^{\circ}$

Fig. 8 Velocity contour at cross section A, S3.

The cylindrical cavity, S1, is the baseline case shown in Fig. 6. Fig. 6 (a-e) are in the ingestion portion of the cycle, while Fig. 6 (f-j) are in the expulsion stroke. In Fig. 6, it can be seen that the vortex pairs flow moving downstream and upstream (Fig. 6(a)) at different phase angles in one working cycle, exhibiting a clear jet formation as indicated by the expelled vortex. The vortex pair e1 and $\mathrm{c} 1$ from the previous working cycle flow along the centerline in the external region downstream and in the cavity upstream, are followed by the new vortices e2 and c2 generated in this working cycle.

In Fig. $6(\mathrm{a}), \theta=10^{\circ}$ corresponded to the commencement of the upward motion of the diaphragm modeled here by the oscillatory velocity boundary condition at the bottom of the cavity. It coincides with the start of the ingestion stroke by the suction of ambient fluid into the cavity. Fig. 6(a) shows remnants of the previous vortex pairs in the external region and cavity, caused by the previous expulsions and ingestions of the synthetic jet. It also shows the presence of corner vortices at the nozzle entrance. At $\theta=46^{\circ}$. Fig. 6 (b), the new vortex pair rolls up at the entrance of the nozzle and its size is of the order of the nozzle width. Separation of the shear layer near the nozzle exit is also readily visible. When the roll-up process is nearly completed at the ingestion phase of $82^{\circ}$, Fig. $6(\mathrm{c})$, the mixing of the primary vortex pair is mostly completed. Then with the upward motion of the diaphragm, the ingestion velocity in the nozzle deceases gradually at $\theta=118^{\circ}$ and $154^{\circ}$ in Fig. $6(\mathrm{~d})$ and (e). The vortex pair inside the cavity starts to grow in size while it moves into the jet cavity and the ingestion phase is completed. 
The expulsion phase commences at $\theta=190^{\circ}$, Fig. 6(f), with the start of the downward motion of the diaphragm. At $\theta=226^{\circ}$. Fig. $6(\mathrm{~g})$, the shear layer separation occurs near the nozzle entrance, and the expulsion initiates the formation of a vortex pair at the nozzle exit. At $\theta=262^{\circ}$, Fig. $6(\mathrm{~h})$, approaching the maximum expulsion velocity, the vortex pair detaches from the nozzle exit plane and grows in size as it advects downstream. After $\theta=334^{\circ}$ in Fig. 6(i), the expulsion phase will complete and the ingestion stroke will commence again, by which time the vortex pair has advected sufficiently downstream that it is not affected by the suction of ambient fluid into the cavity.

For S2 and S3, similar vortex pairs could be observed in the external regions in Fig. 7 and Fig. 8, however, the vortex pairs in the cavities could not be clearly found, particularly for the contraction shaped cavity. As well, the velocity magnitude decreased sequentially from the cylindrical $(6 \mathrm{~m} / \mathrm{s})$ to conical $(5 \mathrm{~m} / \mathrm{s})$ to contraction $(4 \mathrm{~m} / \mathrm{s})$ cavity with the same inlet velocity boundary condition. At $\theta=262^{\circ}$, the approximate point of maximum expulsion velocity for each shaped cavity, the gradient of velocity at the nozzle entrance for S3 is the smoothest (Fig. 8(h)), because of its shape and this transitional structure can cause higher flow resistance because by flow-restricting cavity walls.

Due to the small velocity gradient and limited space around the nozzle entrance from the cavity, the velocity varies gradually and the formation of vortex pair is not seen in S2 and S3. The axial length $\left(l_{c}=12 d\right)$ of contraction shaped cavity is the largest in the three cases for keeping the same cavity volume, and the longest wall condition has the largest flow resistance causing the lowest jet velocity for S3.

\section{IV.Conclusion}

Radial profiles of jet velocity at the exit plane demonstrated noticeable differences in the velocity profile throughout the excitation cycle for the three cavity shapes while the velocity curves for S1, S2 and S3 had the same shape, but different magnitudes. The maximum exit flow rate ratio normalized by the maximum inlet flow rate decreased sequentially from the S1 cylindrical (0.404) to S2 conical (0.342) to S3 contraction (0.285) cavity. The momentum comparison implies a momentum loss that needs to be considered when deploying SJAs.

The velocity contour for S1, S2 and S3 demonstrated that the vortex pairs could be observed in all of the external regions, however, internal vortices in the S2 and S3 cavities could not be clearly found, notably for the contraction shaped cavity, S3. Smooth transitional structure from cavity to nozzle caused higher flow resistance because of the flow-restricting cavity walls that caused the formation of vortex pair to be difficult near the nozzle entrance in the cavity. Because of the interaction within the sharp cavity corners with the local recirculating flow, S1 could achieve 
the highest jet velocity and flow rate. The results confirmed that synthetic jet performance depend on cavity shape. From this, if the goal for synthetic jet is to maximize momentum flux, the basic cylindrical shape should be employed, although geometries S2 and S3 may produce lower pressure loss due to the smooth transition.

\section{Acknowledgments}

This work was supported by the Natural Science and Engineering Research Council (NSERC) of Canada and the Southern Ontario Smart Computing Innovation Platform (SOSCIP). Computations were performed on the Sandybridge (Sandy) cluster at the SciNet HPC Consortium which includes support from the Canada Foundation for Innovation under the auspices of Compute Canada, the Government of Ontario, Ontario Research Fund - Research Excellence, the University of Toronto. Additional support was provided by the China Scholarship Council (CSC).

\section{References}

[1] Greenblatt, D., Paschal, K. B., Yao, C. S., and Harris, J., "Experimental Investigation of Separation Control Part 2: Zero MassFlux Oscillatory Blowing,” AIAA Journal, Vol. 44, No.12, 2006, pp. 2831, 2845.

doi: $10.2514 / 1.19324$.

[2] Feero, M. A., Lavoie, P., and Sullivan, P. E., "Influence of synthetic jet location on active control of an airfoil at low Reynolds number," Experiments in Fluids, Vol. 58, No.8, 2017, pp. 99.

doi: 10.1007/s00348-017-2387-x.

[3] Bhapkar, U. S., Srivastava, A., and Agrawal, A., "Proper cavity shape can mitigate confinement effect in synthetic jet impingement cooling,” Experimental Thermal \& Fluid Science, Vol. 68, 2015, pp. 392, 401. doi:10.1016/j.expthermflusci.2015.05.006.

[4] Utturkar, Y., Mittal, R., Rampunggoon, P., and Cattafesta, L., "Sensitivity of synthetic jets to the design of the jet cavity," 40th AIAA Aerospace Sciences Meeting \& Exhibit, Aerospace Sciences Meetings, 2002-0124. doi: $10.2514 / 6.2002-124$.

[5] Jain, M., Puranik, B., Agrawal, A., “A numerical investigation of effects of cavity and orifice parameters on the characteristics of a synthetic jet flow,” Sensors \& Actuators A Physical, Vol.165, No.2, 2011, pp.351, 366. doi: 10.1016/j.sna.2010.11.001.

[6] Perumal, D. A., and Dass, A. K., "Multiplicity of steady solutions in two-dimensional lid-driven cavity flows by Lattice Boltzmann Method," Computers \& Mathematics with Applications, Vol. 61, No.12, 2011, pp.3711, 3721. doi:10.1016/j.camwa.2010.03.053. 
[7] Jahanshaloo, L., Sidik, N. A. C., Fazeli, A., and Mahmoud, P. H. A., “An overview of boundary implementation in lattice boltzmann method for computational heat and mass transfer," International Communications in Heat \& Mass Transfer, Vol.78, 2016, pp.1, 12.

doi:10.1016/j.icheatmasstransfer.2016.08.014.

[8] Geller, S., Krafczyk, M., Tölke, J., Turek, S., and Hron, J., "Benchmark computations based on lattice-boltzmann, finite element and finite volume methods for laminar flows," Computers \& Fluids, Vol.35, No.8-9, 2006, pp. 888, 897. doi: 10.1016/j.compfluid.2005.08.009.

[9] Kotapati, R. B., Mittal, R., and Cattafesta, L. N. I., "Numerical study of a transitional synthetic jet in quiescent external flow," Journal of Fluid Mechanics, Vol.581, 2007, pp.287, 321. doi: $10.1017 / \mathrm{S} 0022112007005642$.

[10] Wu, D. K. L., and Leschziner, M. A., "Large-eddy simulations of circular synthetic jets in quiescent surroundings and in turbulent cross-flow," International Journal of Heat \& Fluid Flow, Vol.30, No.3, 2009, pp.421, 434. doi: 10.1016/j.ijheatfluidflow.2009.01.007.

[11] Lee, C. Y., and Goldstein, D. B., “Two-Dimensional Synthetic Jet Simulation,” AIAA Journal, Vol.40, No.3, 2002, pp.510, 516. doi: $10.2514 / 2.1675$.

[12] Feero, M., Lavoie, P., and Sullivan, P., "Influence of Cavity Shape on Synthetic Jet Performance," Sensors and Actuators A: Physical, Vol. 223, 2015, pp.1, 10. doi: 10.1016/j.sna.2014.12.004.

[13] Holman, R., Utturkar, Y., Mittal, R., Smith, B. L., and Cattafesta, L., "Formation Criterion for Synthetic Jets," AIAA Journal, Vol.43, No.10, 2005, pp.2110, 2116. doi:10.2514/1.12033.

[14] Mu, H. B, Yan, Q. D., Wei, W., and Sullivan, P., "Unsteady simulation of a synthetic jet actuator with cylindrical cavity using a 3-D Lattice-Boltzmann Method," International Journal of Aerospace Engineering, in Press. 